\title{
A rare case of concomitant pneumocephalus and pneumorachis after lumbar spine surgery with late presenting dural leak
}

\author{
Mayank Gupta ${ }^{1} \cdot$ Kalidindi Kalyan Kumar Varma ${ }^{1} \cdot$ Harvinder Singh Chhabra ${ }^{1}$
}

Received: 31 August 2019 / Revised: 30 September 2019 / Accepted: 30 September 2019

(c) International Spinal Cord Society 2019

\begin{abstract}
Introduction We report a case of pneumocephalus and pneumorachis i.e., air in the cranial cavity and the spinal canal, which are rarely associated with a primary spinal cause. Their concomitant occurrence and association with a late presenting dural leak are also uncommon.

Case presentation A 70-year-old man presented 1 month after decompressive surgery for lumbar canal stenosis with leakage of cerebrospinal fluid (CSF) from the wound site and severe headache. There were no signs of surgical site infection or meningitis. There was no intraoperative or immediate postoperative evidence of dural tear. He was neurologically intact. On detailed work-up, he was found to have air collections in the subarachnoid and intraventricular spaces in the brain and intraspinal air in both the cervical and the lumbar regions. He showed gradual resolution of these findings radiologically and symptomatic improvement through conservative management with analgesics, empirical antibiotics, high flow oxygen, and maintenance of hydration within 1 month.

Discussion Pneumocephalus and pneumorachis are commonly seen after trauma. Spine surgery is a rare cause. They may present with symptoms similar to meningitis and may be erroneously diagnosed and treated. No standard treatment guidelines exist in the literature. Most cases have been managed conservatively. Early detection may allow noninvasive management leading to complete resolution.
\end{abstract}

\section{Introduction}

Pneumocephalus and pneumorachis, i.e., the presence of air in the cranial cavity and the spinal canal respectively, are uncommon conditions rarely associated with a primary spinal cause [1]. These may be symptomatic; however, as spine surgeons usually do not encounter them in routine practice; they are difficult to identify and therefore, treat. Only two cases have been reported in the literature presenting with both of these findings concomitantly after lumbar spine surgery. Only one of these had a late presenting dural leak [1,2]. We report such a rare case, only the second in literature, having the peculiarity of association with a late presenting dural leak and the involvement of both cervical, as well as lumbar regions of the spine.

\section{Mayank Gupta}

mynk_vmmc@yahoo.in

1 Department of Spine Surgery, Indian Spinal Injuries Centre, Sector C, Vasant Kunj, Delhi 110070, India

\section{Case presentation}

A 70-year-old man was operated at our institute for degenerative multilevel lumbar canal stenosis at the L4-5 and L5-S1 levels with neurogenic claudication in bilateral lower extremities. A two-level decompressive laminoforaminotomy was done. The intraoperative and postoperative course was uneventful. He had relief in his claudication symptoms and improvement in walking distance. He was discharged on the third postoperative day. He presented to the outpatient department 1 month postoperatively with a profuse discharge of clear watery fluid from the surgical site that started 1 week after his discharge from the hospital and a headache that had been present for 5 days. There was no history of fever, nausea, vomiting, or pain at the surgical site. The leakage was excessive enough to soak the patient's bed sheets and required multiple dressing changes daily. The headache was diffuse, severe, progressive, worse in an upright position, and relieved slightly by lying supine. It was severe enough to disturb his sleep and required roundthe-clock analgesics. He was taking tablet paracetamol $500 \mathrm{mg}$ four times daily for pain. There were no 
Fig. 1 Images showing the presence of pneumorachis at the presentation (marked by white arrows). a T2W sagittal MRI image of the cervical spine showing air in the spinal canal anterior to the spinal cord. b T2W sagittal MRI of the lumbar spine showing intraspinal air in the L5-S1 region. c T2W axial MRI of the L5-S1 region showing intraspinal air. d Axial CT cut at L4-5 level showing intraspinal air bubbles
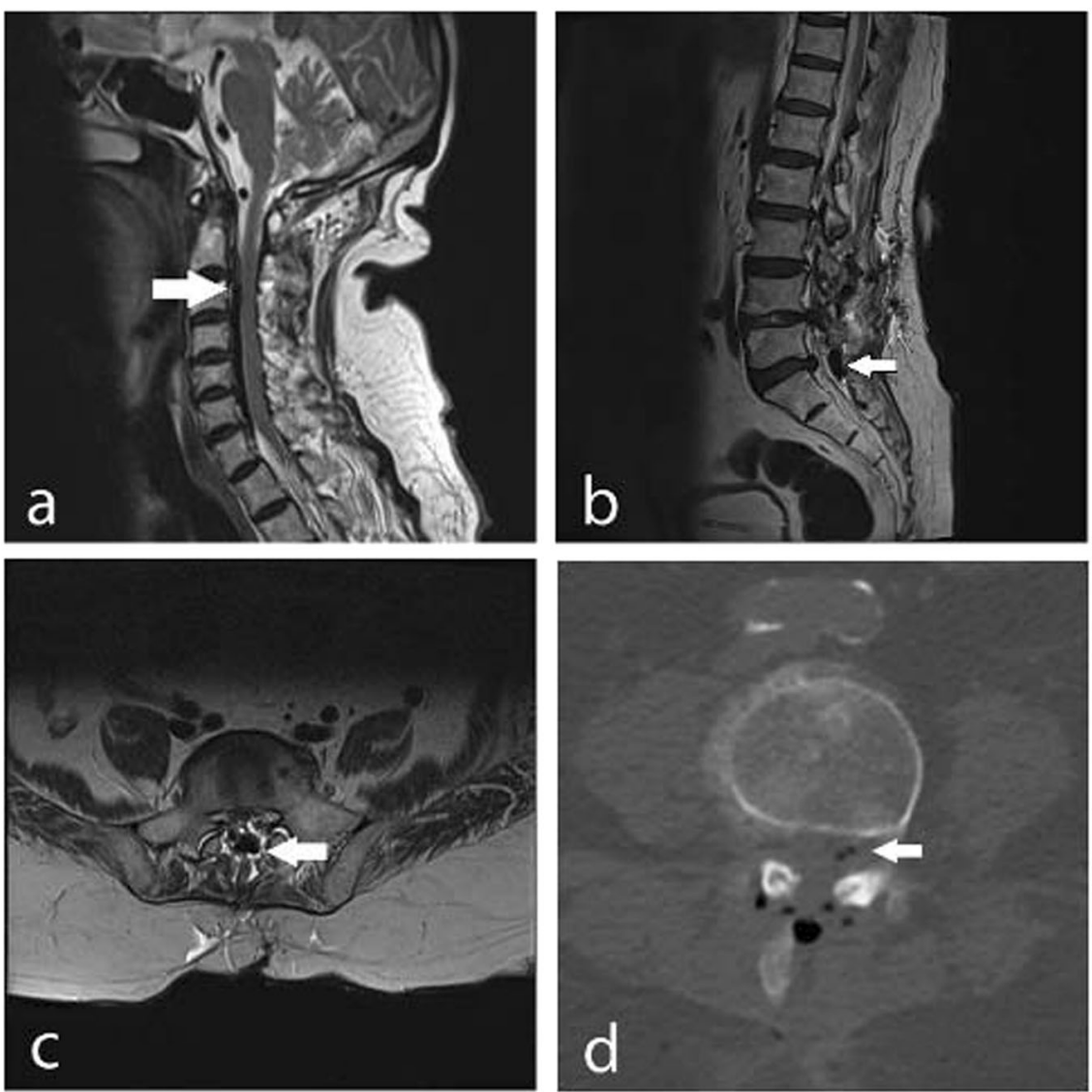

constitutional symptoms. There were no visual or hearing disturbances. The patient had a history of type II diabetes mellitus and hypertension and was on medication for both of these. He was taking tablet atorvastatin $10 \mathrm{mg}$ once daily, tab ecosprin $75 \mathrm{mg}$ once daily, combination of linagliptin $2.5 \mathrm{mg}$ and metformin $500 \mathrm{mg}$ twice daily, and tablet amlodipine $5 \mathrm{mg}$ once daily for these comorbidities.

On examination, the patient was conscious, oriented, and responded well to commands. Higher cognitive functions, cranial nerve function, and cerebellar functions were normal. He was afebrile. There was no neck rigidity or other signs of meningitis. The surgical site showed wound gaping but there were no signs of infection. There was a continuous discharge of clear watery nonfoul-smelling fluid from the surgical site which aggravated on coughing. A clinical diagnosis of cerebrospinal fluid (CSF) leak was suspected.

He was readmitted and a sample of the discharge sent for microbiological investigations. He was started on conservative management consisting of analgesics for headache. Intravenous (i.v.) paracetamol infusion $1 \mathrm{~g}$ twice daily and i.v. diclofenac infusion $75 \mathrm{mg}$ twice daily were started. In addition injection tramadol $100 \mathrm{mg}$ i.v. was given on as and when required basis. Magnetic resonance imaging (MRI) of the lumbosacral spine with whole spine screening was performed which revealed evidence of CSF leakage from the lumbar operated area with a tract communicating with the dura. There was an unexpected presence of air in the intraspinal region in the lumbar spine. Similarly, air was found anterior to the spinal cord in the cervical region. Noncontrast computed tomography and MRI of the brain performed for persistent headache revealed multiple pockets of air in the subarachnoid space in the frontal, as well as temporal regions and also in the intraventricular spaces (Fig. 1). Based on these findings, a diagnosis of pneumocephalus and pneumorachis was made. Neurological examination remained normal. Wound debridement and multi-layered water-tight closure of the wound, with special care of deep fascia, was performed 1 day after admission under local anesthesia. No duroplasty was performed and no drain was used. Empirical intravenous antibiotics were started to prevent meningitis. They were changed to definitive antibiotics based on the CSF culture and sensitivity reports.

Postoperatively, he showed improvement in his symptoms and there was no wound leakage after closure. The headache persisted with similar character as preoperatively. The patient was started on high flow oxygen $(5 \mathrm{l} / \mathrm{min})$ via face mask and adequate hydration was maintained. The wound healed normally and stitch removal was performed 2 weeks postoperatively. The headache improved gradually within 40-45 days after starting treatment and there was a 
resolution in the pneumorachis and pneumocephalus, as documented on MRI, scans done 1 month postoperatively (Fig. 2). The patient was asymptomatic at 2 years after index surgery and is still under follow-up.

\section{Discussion}

CSF leakage after an incidental durotomy is one of the most common complications of spine surgery with a documented incidence of 5.5-9\% for primary lumbar spine surgeries [3]. Camissa et al. reported an incidence of symptomatic delayed dural leaks of $0.28 \%$ in patients (Fig. 3) with no intraoperative or immediate postoperative evidence of incidental durotomy [4]. However, the true incidence of unrecognized durotomies is unknown as most cases are asymptomatic [4]. Routinely encountered complications of CSF leakage are meningitis, CSF fistula, and pseudomeningocoele formation [3]. Pneumocephalus and pneumorachis are extremely rare complications of CSF leakage [1].

Though a few cases of pneumocephalus after spine surgery have been reported, only one case report of pneumorachis after an incidental durotomy has been documented in the existing literature $[1,5]$. The presence of both pneumocephalus and pneumorachis after primary spine surgery is even more rare with only two cases reported till date $[1,2]$. In a case report by Karavelioglu et al. [1], these complications were recognized in the immediate
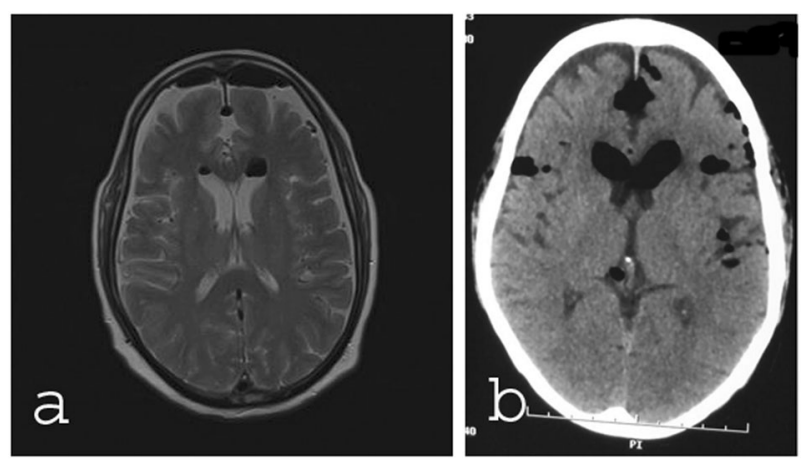

Fig. 2 Images showing pneumocephalus in the subarachnoid and intraventricular spaces of the brain in a T2W axial MRI and b CT scan postoperative period on day 1 with symptoms of pneumocephalus but pneumorachis was asymptomatic. In addition the pneumorachis was only localized to the lumbar spine and the case was managed conservatively.

Akyuz et al. [2] found these complications in a patient who presented two and a half months postoperatively with symptoms suggestive of CSF leakage and meningitis. The case was managed surgically with duroplasty because of meningitis not responding to antibiotics and not primarily for pneumocephalus. The dural tear was unrecognized intraoperatively in both the above cases.

The rarity of association of pneumocephalus and pneumorachis after an uneventful spine surgery justify reporting of our case. The association with a late presenting dural leak makes it extremely uncommon to encounter such a case in routine practice.

Pneumocephalus has been reported mostly after trauma, infections, neoplasms, dural puncture, and rarely after spinal surgery $[1,6]$. It can be located in epidural, subdural, subarachnoid, intraventricular or intraparenchymal spaces [1].

Pneumorachis has been reported commonly after injury to the respiratory system, cranial injuries, or injury to abdominal viscera [5]. Spinal causes are rare with reported etiologies being a vacuum herniated disc, epidural abscess, dural tear, and lumbar puncture [5]. Pneumorachis may be intradural or extradural [5]. It usually presents in the cervical region [5]. Involvement of both cervical and lumbar regions, as in our case, is very rare.

Presence of air in the cranial cavity presents mostly with headache and other nonspecific symptoms like dizziness, lethargy, nausea, and vomiting [1]. Pneumorachis, by contrast, is usually asymptomatic [5]. There may be associated fever or neck rigidity suggestive of coexisting meningitis.

The inverted soda-bottle effect has been described as the mechanism of these complications [7]. As the CSF flows out, the air enters the dural rent through communication with the exterior to equilibrate the pressure by filling in the empty spaces. Due to the upright position of the patient, it usually travels to the intracranial and cervical regions.

No standard treatment guidelines could be found regarding the management of these complications due to the paucity of literature regarding the management of these
Fig. 3 Images showing resolution of pneumocephalus and pneumorachis after 1 month of presentation in (a) T2W axial MRI of the brain, (b) T2W sagittal MRI of the cervical spine, and (c) T2W sagittal MRI of the lumbar spine
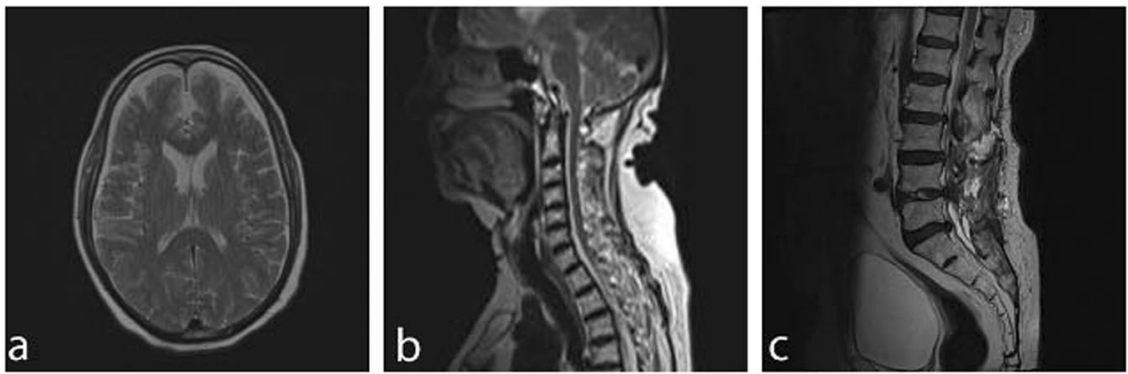
complications, most of them being case reports. The majority of reported cases have been managed successfully with conservative treatment. Once the communication with the exterior is healed, the air gradually is displaced with oxygen which later gets resorbed [7]. Turgut et al. quoted that $85 \%$ of the cases of pneumocephalus resolve spontaneously within 2-3 weeks with conservative management consisting of analgesics for headache, inhalation of $100 \%$ oxygen, and maintenance of hydration [8]. The conservative management for pneumocephalus showed good results in our case as well. Likewise, pneumorachis also responds well to conservative management [5]. Water-tight closure and maintenance of head-down position for few days after an intraoperatively identified lumbar incidental durotomy may help in preventing these complications [1]. Coexisting meningitis, if present, needs to be addressed concomitantly [1]. Antibiotic prophylaxis should be instituted if there is evidence of CSF leakage from the surgical wound to prevent meningitis [1].

In sum, we report a case of late presenting dural leakage after lumbar spine surgery complicated with concomitant pneumocephalus and pneumorachis involving both the cervical and lumbar spine. The extreme rarity of the condition merits publication. As the literature on such cases is limited, standard guidelines do not exist on the management. Identification, as well as reporting of more cases and high quality studies, may help to formulate definitive management guidelines for these entities in the future.

Acknowledgements The authors would like to thank the patient and his family to give consent for reporting of this case.

\section{Compliance with ethical standards}

Conflict of interest The authors declare that they have no conflict of interest.

Publisher's note Springer Nature remains neutral with regard to jurisdictional claims in published maps and institutional affiliations.

\section{References}

1. Karavelioglu E, Eser O, Haktanir A. Pneumocephalus and pneumorrhachis after spinal surgery: case report and review of the literature. Neurol Med Chir (Tokyo) 2013;54:405-7. https://doi.org/ 10.2176/nmc.cr2013-0118.

2. Akyüz O, Gökpınar D, Aydın E, et al. Pneumocephalus and pneumorrhachis after spinal surgery. Pol J Radiol 2016;81:34-5. https://doi.org/10.12659/PJR.895570.

3. Menon SK, Onyia CU. A short review on a complication of lumbar spine surgery: CSF leak. Clin Neurol Neurosurg 2015;139:248-51. https://doi.org/10.1016/j.clineuro.2015.10.013.

4. Cammisa FP Jr, Girardi FP, Sangani PK, Parvataneni HK, Cadag S, Sandhu HS. Incidental durotomy in spine surgery. Spine 2000;25:2663-7.

5. Chaichana KL, Pradilla G, Witham TF, Gokaslan ZL, Bydon A. The clinical significance of pneumorachis: a case report and review of the literature. J Trauma 2010;68:736-44. https://doi.org/10.1097/ TA.0b013e3181c46dd3.

6. M Das J, Bajaj J. Pneumocephalus. StatPearls. Treasure Island (FL): StatPearls Publishing. 2019.

7. Ozturk E, Kantarci M, Karaman K, Basekim CC, Kizilkaya E. Diffuse pneumocephalus associated with infratentorial and supratentorial hemorrhages as a complication of spinal surgery. Acta Radiol 2006;47:497-500.

8. Turgut M, Akyüz O. Symptomatic tension pneumocephalus: an unusual post-operative complication of posterior spinal surgery. $\mathrm{J}$ Clin Neurosci 2007;14:666-8. 